\title{
Contaminazioni
}

\author{
Maurizio Fea
}

\section{Formiche e pachidermi}

Un recente articolo pubblicato su BMJ luglio 2016 dal titolo "Conflict of interest between professional medical societies and industry: a cross-sectional study of Italian medical societies' websites" rivela in modo documentato e inoppugnabile, quello che da tempo tutti sappiamo, ovvero che i rapporti tra industria del farmaco e società scientifiche sono intrecciati e densi e che poche società sembrano preoccuparsi dei potenziali effetti di condizionamento sulla attività clinica e di ricerca, che questi rapporti potrebbero esercitare.

La ricerca ha analizzato i siti web di 154 società iscritte alla Federazione Italiana Società Medico Scientifiche - FISM -, e mostra un quadro in cui solo il 4,6\% delle società possiede un codice etico specifico, il $45,6 \%$ possiede uno statuto che menziona il conflitto di interessi e il $6,1 \%$ ha un bilancio societario trasparente.

Rispetto alla sponsorizzazione industriale, I'indagine evidenzia che il $29 \%$ delle società presenta loghi di case farmaceutiche o di ditte di dispositivi medicali sulla propria pagina web/(home page); inoltre il $67,7 \%$ delle società ha accettato sponsorizzazioni industriali in occasione dell'ultimo congresso (2013/2014).

Il finanziamento dell'industria ai congressi e la presenza di simposi satelliti sponsorizzati riguardano circa i due terzi delle società medico-scientifiche incluse nello studio.

Gli autori concludono che il supporto economico da parte delI'industria, rappresentando una fonte di finanziamento per lo svolgimento di tutte queste attività, può compromettere l'indipendenza della ricerca e dell'aggiornamento medico, un'informazione imparziale e bilanciata e, di conseguenza, la qualità delle cure e dell'assistenza.

L'interesse primario delle società medico scientifiche rischia così di essere pregiudicato da un interesse secondario.

Un aspetto messo in evidenza dall'articolo è che, paradossalmente, la presenza di un codice etico sul conflitto di interessi sembra associarsi a una maggiore presenza di sponsorizzazioni industriali nel programma dell'ultimo congresso nazionale.

La Federazione degli Ordini dei Medici e Farmindustria, hanno recentemente approvato un accordo, adottando il Codice sulla Disclosure della Federazione Europea delle Associazioni e delle Industrie Farmaceutiche (EFPIA).

L'accordo prevede che dal 30 giugno 2016 sarà pubblicato sui siti aziendali I'ammontare delle erogazioni economiche effettuate nell'anno 2015, nel pieno rispetto della normativa sulla privacy, in forma individuale, per i singoli professionisti che avranno dato il proprio consenso.

Per tutti gli altri i dati verranno resi noti in forma aggregata.

Indubbiamente si tratta di un passo avanti nel rendere meno opaco il rapporto che regola l'attività di società e singoli professionisti sanitari, con fonti di finanziamento importanti come quelli che vengono dalla industria.

Dunque il tema del potenziale conflitto di interesse comincia ad essere preso in considerazione seriamente, non solo per farne materia di denuncia scandalistica, ma per essere affrontato e gestito nei modi e nei luoghi più idonei, cominciando a rendere meno opaco questo rapporto.

Naturalmente questo processo avrà degli effetti e comporterà progressivamente delle conseguenze per tutti i professionisti sanitari, non solo per le società che bene o male li rappresentano.

Ad esempio quanti professionisti sarebbero disposti a pagare un costo di iscrizione societario, o iscrizioni a congressi economicamente adeguati a coprire i costi dell'aggiornamento obbligatorio che viene assicurato tramite congressi, riviste, news letter e altre attività formative?

La domanda non è retorica ed è giunto il momento di porsela seriamente.

Le posizioni morali hanno sempre un costo, grande o piccolo che sia; obbligano alla coerenza nel rispettare i principi che hanno ispirato la posizione morale e talora impongono anche un costo economico.

Come diceva un illustre uomo politico, non chiederti cosa può fare il tuo paese per te ma chiediti che cosa puoi fare tu per il tuo paese, ovvero quanto sei disposto ad impegnarti perché le cose possano cambiare, e più prosaicamente, quanto sei disposto a spendere, o a che cosa sei disposto a rinunciare per far sì che il cambiamento possa avvenire.

Sembra difficile che si possa rinunciare al supporto economico dell'industria e alla diverse possibilità di collaborazione, come ricerca, aggiornamento e formazione, che in tutto il mondo sostengono attività medico scientifiche e istituzioni prestigiose; sarebbe anche poco avveduto privarsi di risorse difficilmente acquisibili in altro modo, per ragioni quali il fatto che il denaro può facilmente alterare i rapporti e corrompere le finalità di una società o di una istituzione e quindi ridurre l'autonomia decisionale o il valore di ciò che viene prodotto grazie a quei finanziamenti.

L'albero si giudica dai frutti che porta e per rimanere su questa metafora evangelica, importante è il concime che si usa, la cura che l'agricoltore vi destina, e le circostanze ambientali che ne possono favorire o compromettere lo sviluppo.

I conflitti potenziali di interesse che possono influire sulla bontà dei frutti, non sono generati solo dalla brama di denaro, ma dalla brama di successo: la carriera professionale e accademica, la notorietà che si acquisisce con ricerche e studi che indirettamente possono procurare anche vantaggi economici, riconoscimenti pubblici e gratificazioni per il proprio narcisismo, posizioni di potere nelle aziende sanitarie, tutti aspetti che sono molto più difficili da controllare e valutare, ma che non dimeno possono esercitare potenti influenze sulle decisioni dei singoli e delle società scientifiche in materia di salute e di cura dei pazienti.

Dunque cosa si può fare per controllare ed evitare che tutte queste situazioni, possano condizionare ed influire negativamente sullo scopo primario delle attività sanitarie prodotte da singoli o da associati, di offrire le migliori competenze e le migliori cure alle persone. 
L'accordo tra Farmindustria e FNOMCeO va certamente nella giusta direzione di rendere trasparenti i rapporti economici che intercorrono tra sanitari e industria, la cui opacità ha permesso talora di fare prevalere gli interessi commerciali ed economici di entrambe le parti, su quelli di salute.

Questo segna un cambiamento importante perché permette di giudicare con la misura della evidenza e non del sospetto, il senso ed il valore di collaborazioni e partnership, che sono allo stato attuale indispensabili per il sistema sanitario e la ricerca biomedica nel suo complesso.

Rendere chiari i rapporti può servire ad attenuare quella cultura del sospetto che miopie moraleggianti e talora interessate alimentano, cercando di demonizzare o mettere in discussione il valore di qualunque progetto di ricerca o formazione, che veda l'azione promozionale o di sostegno da parte della impresa.

Cultura del sospetto che preferisce ricorrere agli anatemi liquidatori o alle sentenze giudiziarie, piuttosto di misurarsi su un tema complesso come il conflitto di interessi, ricercando strategie e passi concreti per controllare le possibili derive che generano cattivo uso di risorse.

Non va neanche dimenticato che conflitti di interesse si pongono anche quando si usano risorse pubbliche per finalità personali che nulla hanno a che fare con la salute delle persone, o quando si sostengono e si finanziano progetti di ricerca o piani di formazione di dubbia qualità.

Dunque, come diceva un altro politico a proposito del finanziamento pubblico dei partiti, tutti colpevoli nessun colpevole?

$\mathrm{Ci}$ auguriamo proprio che il processo avviato segua un'altra strada che cominciando dalla disclosure, affronti via via i numerosi ed intricati aspetti legati alla ricerca e utilizzo di risorse economiche utili per una buona sanità.

Provo ad indicare alcuni nodi che a mio avviso andrebbero affrontati, oltre a quelli che emergono con chiarezza dalle conclusioni del BMJ, che riguardano la necessità di rendere trasparenti i bilanci, di dotarsi di codice etico specifico sulla regolazione dei conflitti, di comitati etici che abbiano l'autorevolezza per disciplinare e gestire i possibili conflitti.

Cominciamo con l'esorbitante numero di società scientifiche che affligge tutte le discipline, chi più chi meno: che cosa giustifica l'esistenza di tutte queste società, sono davvero necessarie, quali interessi rappresentano, cosa producono.

Siamo il paese dei campanili e ognuno pretende la sua dose di riconoscibilità, ma questo non produce forse dispersione di risorse, moltiplicazione di iniziative simili, troppo poca novità reale nel campo della ricerca e troppa attenzione alla promozione dei farmaci o delle tecnologie medicali - non nel caso delle dipendenze, dove le tecnologie per ora sono soprattutto le risorse umane - e quindi a volte sono queste che vengono promosse.

Sembra una brutta cosa dire che ci sono troppe società medico scientifiche, e che vanno selezionate, ma il legame perverso tra interessi commerciali dell'industria e il narcisismo interessato di professionisti sanitari produce guasti ai quali occorre porre rimedio, selezionando con criteri di evidenza ciò che è utile e va sostenuto, anche disciplinando con appositi criteri, le condizioni necessarie a definire la qualità di società medico scientifica. Tentativi in tal senso in anni recenti si sono arenati, un po' per la difficoltà di definire criteri misurabili oggettivamente ma soprattutto perché gli interessi in gioco sono molto forti e il legislatore non è stato in grado di prendere le decisioni necessarie, resistendo alle pressioni lobbistiche e ai suoi stessi interessi di potere. È necessario definire le condizioni necessarie di società medico scientifica, anche per dare peso e valore alla autorevolezza delle stesse, sempre più chiamate in causa a studiare e proporre linee guida sulla disciplina, anche con valore giuridico, come il lavoro della commissione parlamentare sulle linee guida in materia di sanità sembra prevedere.

In altri paesi le cose funzionano così, viene prodotto un accordo sugli standard, magari con lunghi e defatiganti confronti, ma poi ci si attiene e chi non rispetta le regole va fuori mercato.

Un altro punto chiave è quello dell'influenzamento diretto esercitato dalle aziende sui dirigenti sanitari con forte potere prescrittivo e di acquisto, che prescinde e talora confligge con I'attività delle società disciplinari di riferimento.

La disclosure delle risorse erogate ai singoli professionisti potrebbe, se applicata con maggior rigore di quanto previsto per ora, ovvero sottraendo alla foglia di fico della privacy, il diritto all'anonimato dei percettori delle somme, rendere davvero trasparenti e pesabili, i possibili fattori di condizionamento.

Rimanendo il vincolo del consenso alla pubblicazione, il rischio è che si abbia sì un quadro complessivo delle risorse erogate in forma aggregata, ma le responsabilità individuali continuino ad essere poco valutabili.

Servono indubbiamente delle regole, che permettano di valutare e di assegnare dei pesi ai vari fattori influenzanti le decisioni di carattere sanitario non solo perché si tratta di una obbligazione morale di cui ciascuno risponde, ma anche per equilibrare, se necessario, il valore dell'influenzamento sul lavoro delle commissioni esperte che collaborano con le istituzioni sanitarie alla realizzazione di linee di indirizzo e politiche di salute, senza escludere pregiudizialmente chi potrebbe portare solide competenze ed esperienze, in quanto soggetto a potenziali conflitti, molto frequenti in tali contesti.

Parlare di regole in un paese che ha fatto della regola il paradigma della elusione è rischioso, anche perché la tendenza è quella di affidare a terzi, come la magistratura, il compito di riempire un vuoto normativo che la politica non sa o non vuole riempire e spesso quando lo fa lo fa male.

È certamente più impegnativo ma credo anche foriero di migliori risultati, fare la fatica di avviare onestamente il confronto tra interessati, in questo caso società medico scientifiche, impresa, istituzioni sanitarie, quindi non quelle di controllo, che semai intervengono per far rispettare gli accordi, ma non nel disporli. L'obiettivo non è quello di annullare le influenze, irrealistico, demagogico e spesso utilizzato per secondi fini, ma di esplicitarne il peso quando presenti, riconoscendo al contempo il valore e l'utilità di progetti e ricerche -se e quando lo hanno- a prescindere dal fatto che siano stati realizzati con contributi di impresa. Ci sono i modi e i criteri per fare ciò, come il modello e la pratica della peer rewiev insegna, pur con alcuni limiti.

Si tratta di lavorare in questa direzione con onestà, trasparenza e voglia di far funzionare meglio le cose a vantaggio di tutti, impresa, cittadini, professionisti sanitari, istituzioni.

Nel mondo delle società scientifiche noi siamo una formica, ma anche le formiche possono essere fastidiose e indurre animali più grossi a cambiare posizione.

maurizio.fea@gmail.com

Fabbri A., Gregoraci G., Tedesco D. et al. (2016), "Conflict of interest between professional medical societies and industry: a crosssectional study of Italian medical societies' websites", BMJ Open, 6e01124. doi: 10.1136/ bmjopen-2016-011124.

Lettera agli iscritti giugno 2016 - Obiettivo Trasparenza: I'impegno di Farmindustria e FNOMCeO. 